\title{
Spontaneous Vanishing of Coronary Collaterals During Cardiac Catheterization for Acute Myocardial Infarction
}

\author{
Pierce Dupuis ${ }^{\mathrm{a}}$, Zakaria Sheikhaden ${ }^{\mathrm{a}}$, Jacob Stremers ${ }^{\mathrm{a}}$, Ibriham Shah ${ }^{\mathrm{a}}$, Paul Zack ${ }^{\mathrm{a}, \mathrm{b}}$
}

\begin{abstract}
A patient with acute ST segment elevation inferior myocardial infarction underwent urgent cardiac catheterization. Left coronary arteriography demonstrated minor coronary disease with well-defined collateral filling of the dominant right coronary artery. Shortly thereafter, right coronary arteriography demonstrated entirely normal findings, and a repeat left coronary angiogram no longer demonstrated coronary collaterals. The "vanishing" coronary collaterals are consistent with acute myocardial infarction from transient occlusion of the right coronary artery. These findings are consistent with the phenomenon of myocardial infarction in the presence of angiographically normal coronary arteries. The transient coronary occlusion in this patient is likely secondary to spontaneous lysis of intracoronary thrombus or transient coronary vasospasm.
\end{abstract}

Keywords: Coronary; Collaterals; Myocardial infarction

\section{Introduction}

Patients undergoing cardiac catheterization for acute ST segment elevation myocardial infarction (STEMI) are commonly noted to have total or subtotal coronary artery occlusion, with associated angiographically detected coronary collaterals [1]. Following successful coronary intervention and reperfusion of the occluded vessel, the coronary collaterals can no longer be angiographically visualized [2]. We report a case of a patient with acute STEMI who was found to have spontaneous disappearance of angiographically detected coronary collaterals during a cardiac catheterization procedure without coronary intervention.

\section{Case Report}

A 71-year-old woman presented to the emergency department

Manuscript accepted for publication October 10, 2016

${ }^{a}$ McLaren Greater Lansing Hospital, Lansing, MI, USA

${ }^{\mathrm{b}}$ Corresponding Author: Paul Zack, 2134 Hampton Place, Okemos, MI 48864, USA. Email: Paul.Zack@Mclaren.org

doi: https://doi.org/10.14740/jmc2667w following the sudden onset of chest pain. The pain began 30 min prior to arriving in the emergency department and was located in the mid chest area with radiation to the left chest, shoulder and back. The patient had no previous history of known cardiac disease and no previous symptoms of chest pain. During the 2 weeks prior to hospitalization, the patient had noted occasional symptoms of exertional dyspnea, but no symptoms of shortness of breath at rest, orthopnea, or paroxysmal nocturnal dyspnea. She had noted occasional symptoms of palpitations during the past several weeks, but no episodes of lightheadedness, syncope or near-syncope. The patient's cardiac risk factors included a history of hypertension, diabetes mellitus, hyperlipidemia, exposure to tobacco products and a family history of coronary disease. Medications prior to admission included a beta blocker agent, a statin medication, aspirin and metformin. She had no history of surgical procedures in the past.

On presentation to the emergency department, the blood pressure was $109 / 65 \mathrm{~mm} \mathrm{Hg}$, the pulse rate was 88 per minute and the respiratory rate was 18 per minute. The neck was supple, with no jugular venous distention. The lungs were clear to auscultation. On cardiac examination, there was a regular rhythm with soft systolic murmur heard at the left sternal border. On extremity examination, there was no pedal edema and pedal pulses were palpable bilaterally.

The initial 12-lead electrocardiogram (Fig. 1) showed ST segment elevation of the inferior leads, most pronounced in leads III and AVF, which had $2 \mathrm{~mm}$ of elevation and reciprocal ST segment depression of as much as $4 \mathrm{~mm}$ in the precordial leads V2 through V4. A single view chest radiograph showed borderline cardiac enlargement and clear lung fields. Initial laboratory examination included normal electrolyte, creatinine and complete blood count values, with a mildly elevated troponin-I level of $0.074 \mathrm{ng} / \mathrm{mL}$ (reference range: $0.000-0.056$ $\mathrm{ng} / \mathrm{mL}$ ). The patient was brought urgently to the cardiac catheterization laboratory. After obtaining femoral arterial access, a left coronary diagnostic catheter was introduced and left coronary angiography was performed in multiple projections (Fig. 2 ). The left main and non-dominant left circumflex coronary arteries were angiographically normal, while a moderate lesion was present within the proximal anterior descending artery estimated at $50 \%$ diameter narrowing. Well-defined coronary collateral circulation to the right coronary artery was visualized. The collateral vessels arose from the anterior descending artery and resulted in retrograde filling of the dominant right coronary artery to the mid segment of the right coronary artery. A right coronary guiding catheter was then introduced. 


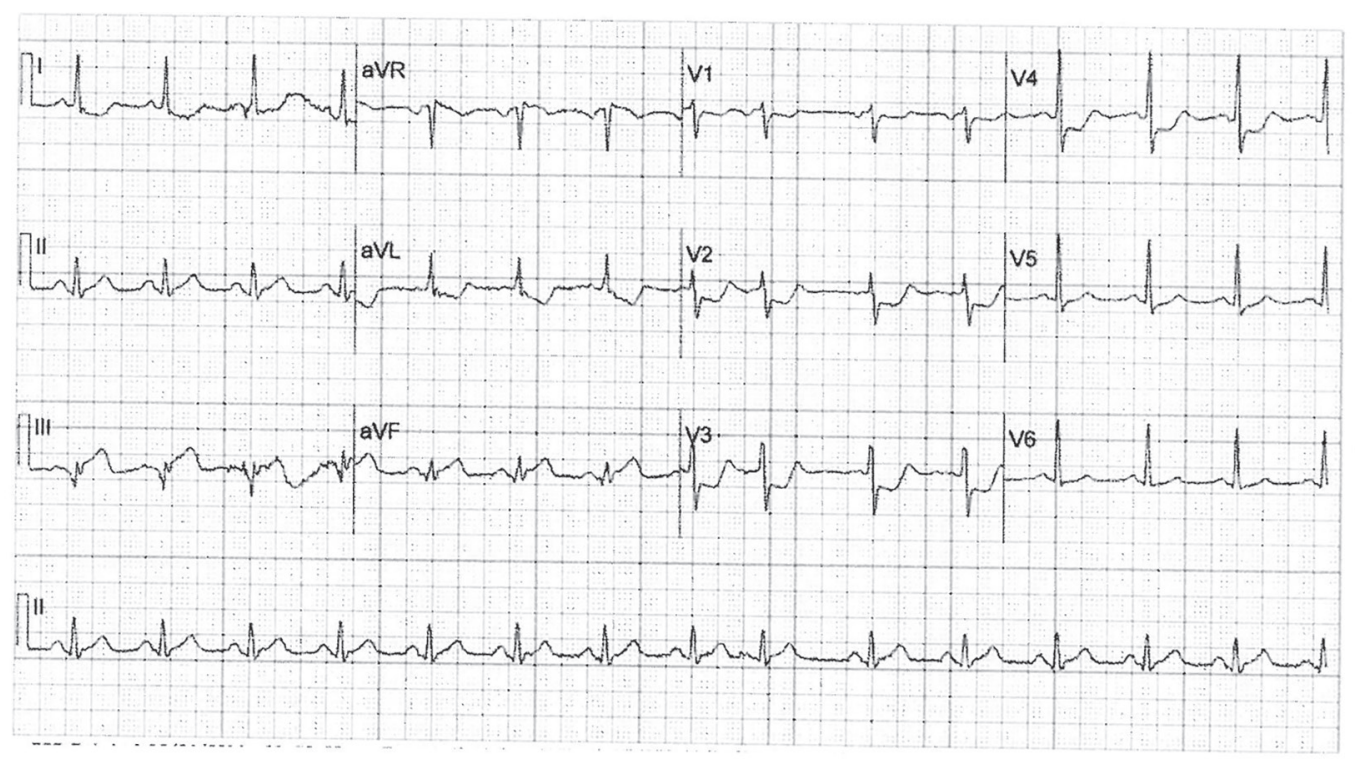

Figure 1. Initial 12-lead electrocardiogram showing ST segment elevation in the inferior leads and reciprocal ST segment depression in the precordial leads.

The right coronary angiogram, performed in multiple projections (Fig. 3), surprisingly revealed an entirely normal dominant right coronary artery, without angiographic evidence of stenosis, plaque or intracoronary thrombus. The left coronary diagnostic catheter was then reintroduced, and the left coronary angiogram was repeated (Fig. 4). The collateral circulation to the right coronary artery was no longer detected. Left ventriculography was then performed in the right anterior oblique projection, utilizing a pigtail catheter. Mild hypokinesis of the inferior left ventricular segment was observed with an estimated ejection fraction of $50 \%$.

The patient was treated with the addition of clopidogrel and amlodipine to the medication regimen, increased doses of statin and beta blocker agents, and continuation of aspirin. She progressed satisfactorily following hospital admission, with no further symptoms of chest pain and no observed arrhythmias on cardiac monitoring. A clinical and laboratory evaluation for collagen vascular abnormalities or hyper-coagulable predisposition was unrevealing.

\section{Discussion}

Our patient's clinical presentation, including findings on electrocardiogram and troponin-I level, was consistent with acute STEMI. The initial left coronary angiogram demonstrated the presence of collateral circulation from the left coronary arterial system to the dominant right coronary artery. It has been demonstrated that the angiographic appearance of coronary collateral vessels is indicative of a $99 \%$ or $100 \%$ coronary artery
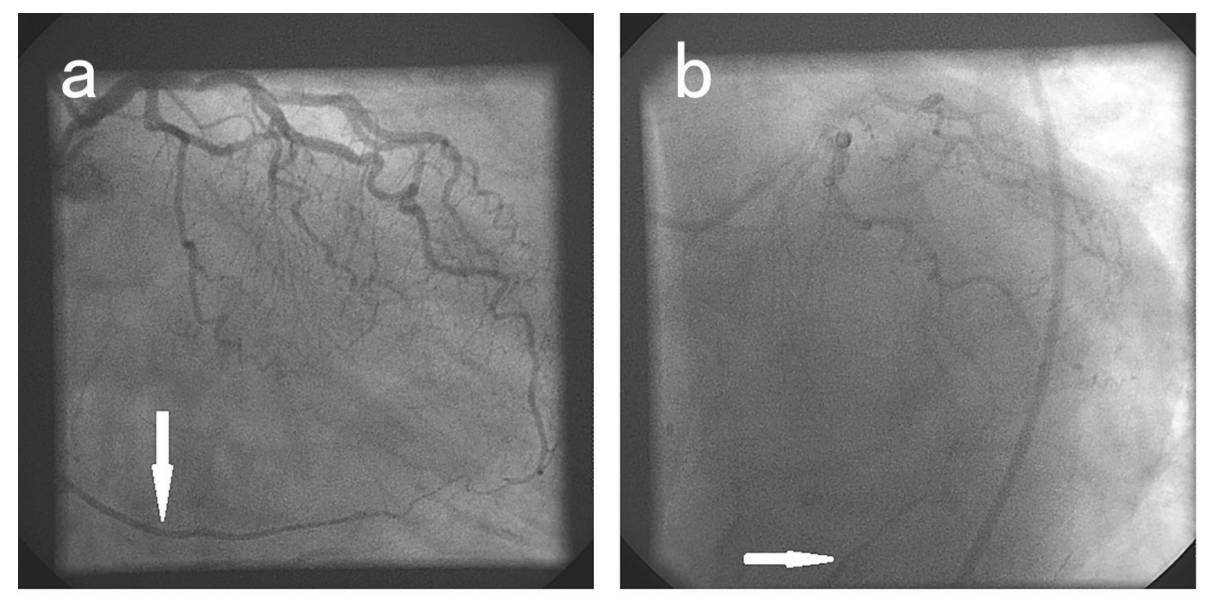

Figure 2. Selective left coronary arteriogram in the right (a) and left (b) anterior oblique projections. Collateral filling of the right coronary artery is seen (arrow). 

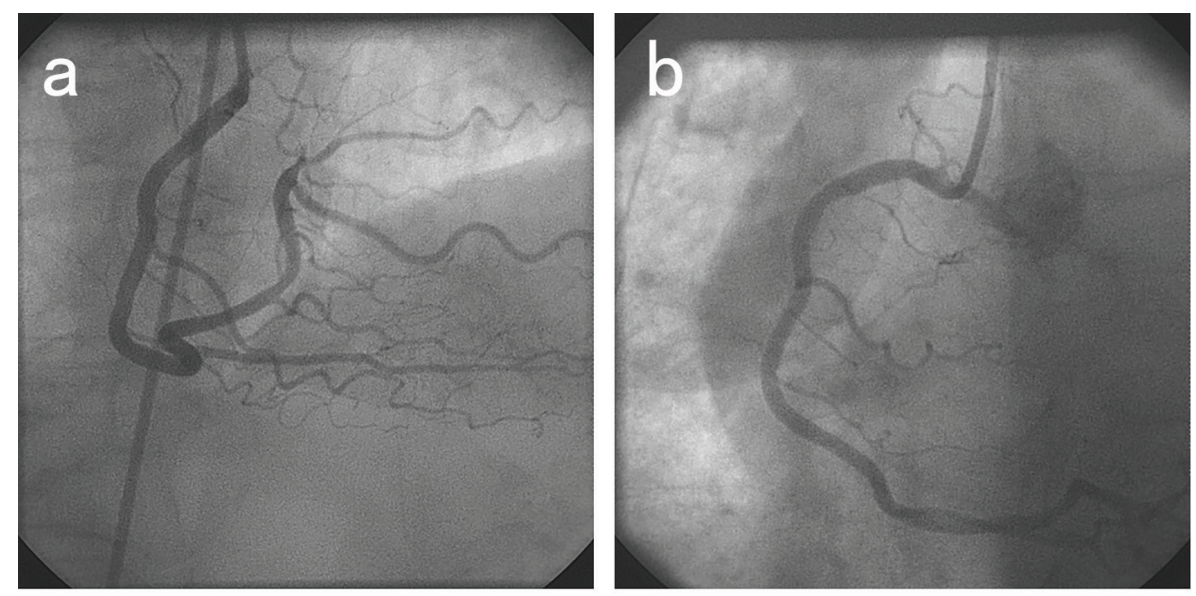

Figure 3. Selective right coronary arteriogram in the right (a) and left (b) anterior oblique projections demonstrating entirely normal findings.

occlusion [3]. It therefore appears evident that, at the time of our patient's left coronary angiogram, the right coronary artery was totally or sub-totally occluded. This suggested right coronary artery occlusion is consonant with our patient's clinical presentation of acute inferior STEMI.

Several minutes after performance of left coronary angiography, selective right coronary angiography demonstrated entirely normal right coronary findings. The absence of right coronary occlusion at this time was confirmed by the subsequent repeat left coronary angiogram, which no longer showed the presence of coronary collateral vessels. It would appear that these "vanishing" coronary collaterals corresponded to the resolution of total or near-total right coronary artery occlusion.

Our patient's acute myocardial infarction in the presence

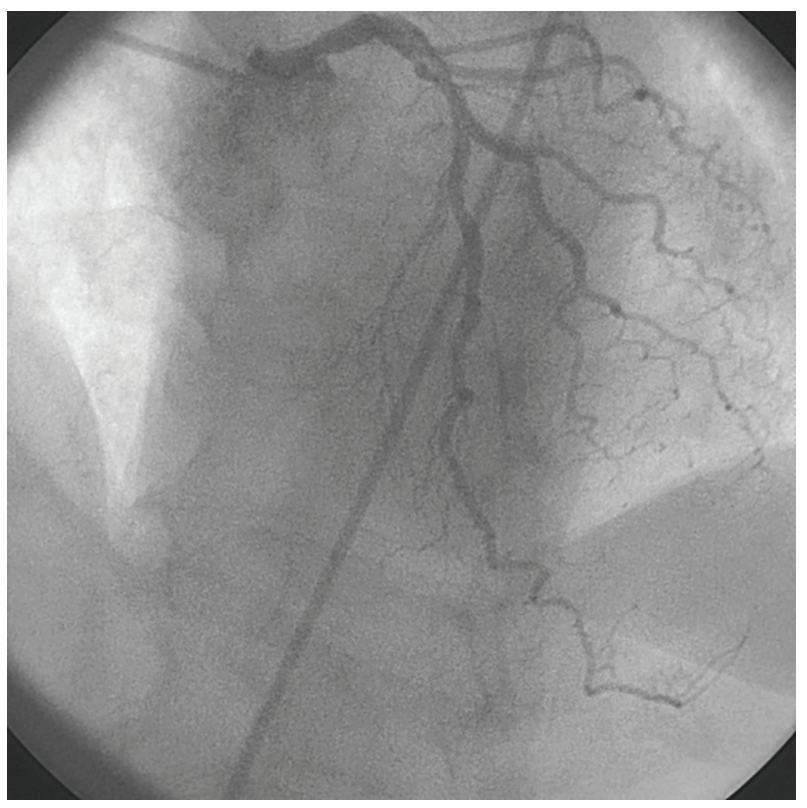

Figure 4. Repeat left coronary arteriogram in the left anterior oblique projection. Collateral filling of the right coronary artery is no longer seen. of a right coronary artery that was normal at the time of selective coronary injection is consistent with the phenomenon of myocardial infarction with angiographically normal coronary arteries. A retrospective analysis of patients with proven myocardial infarction demonstrated that $1.1 \%$ of patients had no luminal irregularities at the time of coronary angiography [4]. The proposed mechanisms for myocardial infarction in the presence of normal coronary arteries include coronary thrombus formation with subsequent spontaneous lysis and coronary vasospasm leading to transient coronary artery occlusion [5]. Both of these processes are compatible with the angiographic findings in our patient. Other reported causes of myocardial infarction without detectable coronary disease, including apical ballooning syndrome, cocaine abuse, viral myocarditis, aortic dissection, and autoimmune vasculitis [6] are inconsistent with our patient's clinical history and findings.

Although the precise etiology of our patient's unusual findings of "vanishing coronary collaterals" remains uncertain, we have elected to continue empiric treatment for both the possibility of transient intracoronary thrombus and coronary vasospasm with a post-hospital discharge regimen that includes a calcium channel blocking agent and dual anti-platelet medication.

\section{References}

1. Tatli E, Altun A, Buyuklu M, Barotcu A. Coronary collateral vessel development after acute myocardial infarction. Exp Clin Cardiol. 2007;12(2):97-99.

2. Berry C, Balachandran KP, L'Allier PL, Lesperance J, Bonan R, Oldroyd KG. Importance of collateral circulation in coronary heart disease. Eur Heart J. 2007;28(3):278291.

3. Elayda MA, Mathur VS, Hall RJ, Massumi GA, Garcia $\mathrm{E}$, de Castro CM. Collateral circulation in coronary artery disease. Am J Cardiol. 1985;55(1):58-60.

4. Ammann P, Marschall S, Kraus M, Schmid L, Angehrn W, Krapf R, Rickli H. Characteristics and prognosis of 
myocardial infarction in patients with normal coronary arteries. Chest. 2000;117(2):333-338.

5. Chandrasekaran B, Kurbaan AS. Myocardial infarction with angiographically normal coronary arteries. J R Soc
Med. 2002;95(8):398-400.

6. Sharifi M, Frohlich TG, Silverman IM. Myocardial infarction with angiographically normal coronary arteries. Chest. 1995;107(1):36-40. 